\title{
Performance of the PTI00 noncontact tonometer in healthy eyes
}

This article was published in the following Dove Press journal:

Clinical Ophthalmology

19 May 201 I

Number of times this article has been viewed

\section{Turki M AlMubrad}

Cornea Research Chair, Department of Optometry, College of Applied Medical Sciences, King Saud University, Riyadh, Kingdom of Saudi Arabia
Correspondence: Turki M AlMubrad, Cornea Research Chair, Department of Optometry, College of Applied Medical Sciences, King Saud University, PO Box 10219, Riyadh II433, Kingdom of Saudi Arabia

Tel +966 I4693555

Fax +966 I4693556

Email turkim@ksu.edu.sa
Background: The purpose of this study was to assess the repeatability and reproducibility of the PT100 noncontact tonometer and to compare its consistency with the Goldmann applanation tonometer (GAT) in measuring intraocular pressure (IOP).

Methods: Triplicate IOP measurements were obtained on two separate occasions using the PT100 and GAT from randomly selected eyes in 66 healthy volunteers aged $22 \pm 1$ years. The repeatability and reproducibility of each techniques was assessed. Agreement between the techniques was statistically quantified using intrasession repeatability for each technique as the basis for comparison.

Results: Both techniques returned equal IOP values in the first measurement session $(15 \pm 3 \mathrm{mmHg})$. The second session showed a mean difference in average IOP $(1 \pm 0.71)$. The $95 \%$ limits of agreement between the techniques were -5.2 to $5.5 \mathrm{mmHg}$ and -4.0 to $4.7 \mathrm{mmHg}$ (sessions 1 and 2, respectively). These mean differences were not statistically significant $(P>0.05$, paired $t$-test), with the PT100 underestimating IOP measurement by $1.00 \mathrm{mmHg}$. The mean intrasession IOP for GAT sessions 1 and 2 was $0 \pm 0.90 \mathrm{mmHg}$ and $0.04 \pm 1.06 \mathrm{mmHg}$, respectively, and the corresponding mean IOP measurement difference for the PT100 was $-0.06 \pm 0.96$ and $-0.39 \pm 0.94 \mathrm{mmHg}$ (sessions 1 and 2, respectively; $P>0.05$, paired $t$-test). Repeatability coefficients for the GAT IOP measurements were $1.8 \mathrm{mmHg}$ and $2.1 \mathrm{mmHg}$ for sessions 1 and 2, while the PT100 repeatability coefficient was $1.9 \mathrm{mmHg}$ and $1.8 \mathrm{mmHg}$ for sessions 1 and 2, respectively. The intrasession repeatability coefficient of both techniques for test-retest differences were within $\pm 5 \mathrm{mmHg}$.

Conclusion: The PT100 noncontact tonometer produced greater repeatability than the GAT in assessment of IOP, whereas GAT resulted in more reproducible results. Both techniques showed a close level of agreement on comparison, with the PT100 underestimating IOP measurement by $1.0 \mathrm{mmHg}$ only, although this was not clinically or statistically significant. Of importance is that the IOP measurements using these techniques could be interchangeable in the IOP range studied here.

Keywords: Goldmann applanation tonometer, intraocular pressure, Reichert PT100, noncontact tonometer, repeatability, reproducibility

\section{Introduction}

Intraocular pressure (IOP) measurement is a compulsory routine procedure in a complete eye care examination, and determination of IOP is essential in the evaluation of ocular health, because it is the main risk factor for developing glaucoma. The Advanced Glaucoma Intervention Study ${ }^{1}$ reported that decreasing the IOP in patients with glaucoma to $<20 \mathrm{mmHg}$ is beneficial, but noted it may not be enough to preserve the visual field, because some patients require a further decrease in IOP to $<18 \mathrm{mmHg}$ 
to avoid visual field damage. The Collaborative Initial Glaucoma Treatment Study ${ }^{2}$ found a significantly reduced loss of visual field if the IOP is kept to $<18 \mathrm{mmHg}$, ${ }^{2}$ affirming that a decrease in IOP delays visual field damage caused by glaucoma.

The Goldmann applanation tonometer (GAT) is currently the most widely used instrument for measuring IOP, ${ }^{3}$ and is considered the "gold standard". ${ }^{4,5}$ However, the GAT has two disadvantages. First, the instrument probe must come into direct contact with the cornea, which can increase the risk of infection. Second, use of the GAT requires a local anesthetic, and some patients, especially children, are unwilling or unable to tolerate drug instillation. With these factors in mind, several noncontact tonometers have been developed to facilitate measurement of IOP during vision screening.

Recently, several portable noncontact tonometers have been developed, ie, the TGDc-01 (Ryazan, Russia), the ICARE tonometer (TA01, Helsinki, Finland), the Tonopen (Mentor, Santa Barbara, CA), and the PT100 tonometer (Reichert Ophthalmics Inc, Depew, NY). The PT100 noncontact tonometer is a battery-operated hand-held portable tonometer weighing approximately $1.3 \mathrm{~kg}$. Because of the key role of tonometry in glaucoma screening and the periodic need to assess IOP outside the clinic, these portable devices have found their usefulness in the heart of active ophthalmology and in optometry practices where nonmedical personnel take IOP measurements.

Several comparative studies have demonstrated the reliability and accuracy of IOP measurements obtained with noncontact tonometers (both desktop and portable) and their correlation with measurements obtained with the GAT in subjects with and without glaucoma. ${ }^{3-9}$ Only two studies have reported comparable IOP measurements with the PT100 noncontact tonometers and the GAT. ${ }^{10,11}$ The study by Salim et al was a comparative study of the PT100 and the GAT, but there was a large age variation among the recruited subjects, which may have affected the accuracy of the results. Murase et al focused on the effect of central corneal thickness on IOP measurements obtained by the PT100 and the GAT. However, neither the Salim nor the Murase study was able to verify the repeatability and reproducibility of IOP measurements in normotensive subjects obtained by the PT100 noncontact tonometer when compared with the "gold standard" of GAT. Therefore, the purpose of this study was to assess further the reliability, repeatability, and reproducibility of IOP measurements obtained with the PT100. We also verified the correlation of IOP measurements obtained by the PT100 and the GAT.

\section{Subjects and methods}

The study participants were 66 patients (46 men, 20 women) aged 20-25 (22 \pm 1$)$ years, who presented for routine oculovisual examinations at the King Saud University optometry clinics.

The study protocol was designed according to the principles of the Declaration of Helsinki and was approved by the ethics committee of the College of Applied Medical Sciences. After verbal instruction about the procedures involved in data collection, all subjects gave their informed consent to participate in this study.

IOP measurements were obtained only from one randomly selected eye of each subject. Randomization was carried out using random numbers generated on a Microsoft Excel spreadsheet. Exclusion criteria were a positive history of ocular disease, systemic disease with ocular implications, ocular surgery, total astigmatism which exceeded $3 \mathrm{D}$, or participation in a similar study carried out earlier at the same institution. A comprehensive ocular examination was carried out to screen each patient for eligibility. No subject was excluded from the study, but seven subjects were lost to follow-up.

For the assessment of IOP using the Reichert PT100 noncontact tonometer, measurements were made in triplicate and averaged to get the IOP reading for one eye. GAT measurements were made in triplicate and averaged to get the IOP for the selected eye. The period of corneal contact with the applanation probe was approximately five seconds (each time) to minimize simultaneously the effects of aqueous massage on repeated applanation readings ${ }^{11,12}$ and the possible errors caused by oscillatory fluctuations in IOP, due mainly to the ocular pulse and respiratory cycles. ${ }^{13-16}$

Noncontact tonometry readings were always made before applanation with the GAT to eliminate the possible effect of ocular massage, which has been reported with the GAT but found to be absent with noncontact tonometers. ${ }^{15-19}$

Repeatability was assessed using all IOP measurements for each technique per subject. Reproducibility was assessed by a re-evaluation of each subject's IOP during a second measurement session taken approximately one week later. The average IOPs for both sessions using one method were compared to assess the reproducibility of that method. The agreement between both techniques was statistically quantified using the repeatability (for each technique) as the basis for comparison.

The data for this study were masked so that the clinician (TA) who performed the GAT never knew the IOP of a subject before performing the procedure on him/her, and the IOP data records were maintained by another clinician, who also 
performed noncontact tonometry using the PT100 in the first session. In the second measurement session, these roles were switched. Each time a Goldmann IOP measurement was made, it was read off the scale by the second clinician, who then reset the scale to an arbitrary zero between 7 and $10 \mathrm{mmHg}$. This procedure was adopted to avoid any tactile clues which might aid the tonometrist to determine the endpoint of a reading. ${ }^{3}$

All IOP measurements were made between 2 pm and 4 pm to ensure that the IOP, although still susceptible to diurnal fluctuations, was assessed at the time of the day when it is most stable. ${ }^{20}$

\section{Statistical analysis \\ IOP measured with PTI00 versus GAT}

The limits of agreement ${ }^{23}$ between the PT100 and the GAT were assessed. To determine the presence or absence of a statistically significant difference between the IOP values returned by both methods, the average IOP using the PT100 was compared with the average IOP using the GAT for each subject at each measurement session. To ensure that each method was consistent and to measure the consistency of the PT100 noncontact tonometer against that of the GAT, the within-session differences for the noncontact tonometer in the first and second measurement sessions and the within-session differences for the GAT in both sessions were compared with each other in other columns (repeated-measures one-way analysis of variance).

\section{Repeatability}

Both tonometers were assessed for repeatability using all three readings for each subject to generate an average IOP and a mean difference. The column of the differences was plotted against the column of average IOPs to determine the limits of repeatability for each technique. Using the formula of Bland and Altman, ${ }^{23}$ the repeatability coefficient $\left(1.96 *\right.$ standard deviation $\left.[\mathrm{SD}]^{\text {within-session differences }}\right)$ was computed twice for each technique (once per session).

\section{Reproducibility}

The difference between the mean IOP in sessions 1 and 2 was determined for each subject, as was the average IOP between sessions. The column of differences was plotted against the column of averages to determine the limits of reproducibility for each technique. The reproducibility of the PT100 was compared with that for the GAT (paired $t$-test) to assess the reproducibility of IOP measured by the PT100. Using the formula of Bland and Altman, ${ }^{23}$ the between-session reproducibility coefficient $\left(1.96 * \mathrm{SD}^{\text {between-session differences }}\right)$ was computed once for each technique. To determine if there was a significant variation in IOP for the subjects between the two measurement sessions, the average IOPs for each subject, measured by both methods, in session 1 were compared with the average IOP measured in session 2 (four columns) using repeated-measures one-way analysis of variance.

For each paired comparison of averages, a paired comparison was also performed for the corresponding withinsubject standard deviations to determine if any potential difference between averages could be explained by a greater variability of the within-subject standard deviations of either average.

Calculations based on a pilot sample of 14 subjects suggested that the number of subjects necessary to achieve a statistical power of $95 \%$, at a statistical level of significance of $5 \%$, was 61 . The sample size calculation was determined from an initial sample of 15 subjects using the statistical freeware $\mathrm{G}^{*}$ Power (version 3.0.5), downloaded from the Internet and used as described elsewhere. ${ }^{23}$ Statistical analyses were made using the Graphpad Instat for Windows program, version 3.00 (Graphpad Software Inc, San Diego, CA, www.graphpad.com).

\section{Results}

The mean subject age was $22 \pm 1$ (range 20-25) years. There were $46(69.7 \%)$ men and $20(30.3 \%)$ women in the study group.

\section{IOP measurement by PTI 00 versus GAT}

The mean IOP readings, SDs, range of values, and repeatability coefficients obtained by each technique in the two measurement sessions are described in Table 1. While both techniques returned equal IOP values in the first measurement session, there was a mean difference in the average IOP at the second measurement session $(1 \pm 0.71)$. These mean differences were not statistically significant $(P>0.05$, paired $t$-test), with the PT100 underestimating the IOP measurement by $-1.00 \mathrm{mmHg}$.

Table I Intraocular pressure values and difference between the tested techniques in $\mathrm{mmHg}$

\begin{tabular}{lllll}
\hline & Total & GAT & PT I00 & GAT-PT I00 \\
\hline Mean \pm SD & 66 & $15 \pm 3.0$ & $15 \pm 3.0$ & $-0.3 \pm 1.2$ \\
Range & 66 & $8-24$ & $9-24$ & $-4+3$ \\
$95 \% \mathrm{Cl}$ & 66 & $13.93-15.56$ & $13.79-15.46$ & $-2.7+2.1$ \\
$\mathrm{R}$ & 66 & $1.8 \& 2.1$ & $1.9 \& 1.8$ & $5.3 \& 4.3$ \\
\hline
\end{tabular}

Abbreviations: $\mathrm{Cl}$, confidence interval; GAT, Goldmann applanation tonometer; PTI00, noncontact tonometer; R, repeatability coefficient session I and session 2; $\mathrm{SD}$, standard deviation. 
The limits of agreement between the techniques are shown in Figure 4. The coefficient of agreement between the techniques was $7.5 \mathrm{mmHg}$ for the first measurement session and $6.1 \mathrm{mmHg}$ for the second measurement session. About $97.2 \%$ of the mean IOP measurements obtained by both instruments were in agreement by $\leq 3 \mathrm{mmHg}$ on intersession analysis.

In the comparison of six columns of differences, there was no statistically significant difference $(P>0.05$, repeated measures one-way analysis of variance). The six columns computed were the column of differences for the PT100 in sessions 1 and 2, the column of differences for the GAT in sessions 1 and 2, and the between-technique difference in sessions 1 and 2 .

\section{Repeatability and reproducibility}

The intrasession IOP mean difference for GAT sessions 1 and 2 was $0 \pm 0.90 \mathrm{mmHg}$ and $0.04 \pm 1.06 \mathrm{mmHg}$, respectively. The corresponding mean difference in IOP measurement for PT100 sessions 1 and 2 was $-0.06 \pm 0.96$ and $-0.39 \pm 0.94 \mathrm{mmHg}$, respectively. The variation between the intrasession difference columns was not statistically significant $(P>0.05$, paired $t$-test).

When repeated, the repeatability coefficients for the GAT and PT100 noncontact tonometer IOP measurements were quite close, as shown in Table 1. A Bland-Altman analysis for the two measurement sessions using the 66 subjects returned an estimated bias with the GAT and the PT100 noncontact tonometer of $0.49 \mathrm{mmHg}$ ( $95 \%$ confidence interval, -0.36 to 1.61 for both techniques) with $95 \%$ limits of agreement of -2.0 to 2.1 and -2.2 to 1.8 . The repeatability differences in IOP as assessed with the PT100 did not vary significantly from those of the GAT ( $P>0.05$, paired $t$-test).

The repeatability coefficient for test-retest differences using both techniques was within $\pm 5 \mathrm{mmHg}$ for both the GAT and the PT100 noncontact tonometer (Figure 3).

In assessing the reproducibility of the two techniques when measurements were taken by two different observers, the reproducibility coefficients in mean difference for both the GAT and the PT100 noncontact tonometer on a Bland-Altman plot was $-0.115 \mathrm{mmHg}$, which did not vary significantly $(P>0.05$, paired $t$-test). There was no significant correlation ( $P>0.05$, Pearson's $r)$ between the magnitude of IOP measurements within sessions for the same technique (Figures 1 and 2), between sessions for the same technique (Figure 3), and between techniques (Figure 4).

The within-subject standard deviation derived from a comparison of every paired comparison of averages revealed the variation was insignificant $(P>0.05$, one-way analysis of variance).

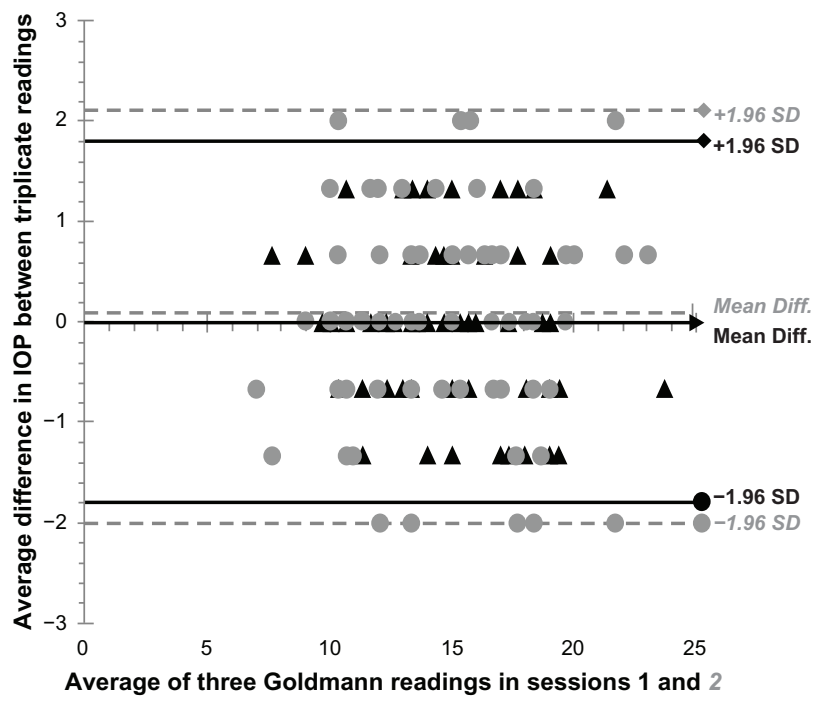

Figure I Intrasession variation of IOP with the Goldmann applanation tonometer in sessions I and 2. The mean difference of triplicate IOP measurements for each subject; $95 \%$ limits of agreement are depicted by the three lines.

Abbreviations: IOP, intraocular pressure; SD, standard deviation.

\section{Discussion}

The Reichert PT100 noncontact tonometer has a repeatability coefficient similar to that of the GAT. When both instruments are used to measure IOP in the same individual, there is a $95 \%$ chance that the difference between the two instruments will not exceed $1 \mathrm{mmHg}$. The repeatability coefficients found in our study were $\pm 1.8 \mathrm{mmHg}$ and $\pm 2.1 \mathrm{mmHg}$ for the GAT (sessions 1 and 2), and $\pm 1.9 \mathrm{mmHg}$ and $+1.8 \mathrm{mmHg}$ for sessions 1 and 2 for the Reichert PT100 noncontact tonometer (sessions 1 and 2). Although the repeatability coefficient

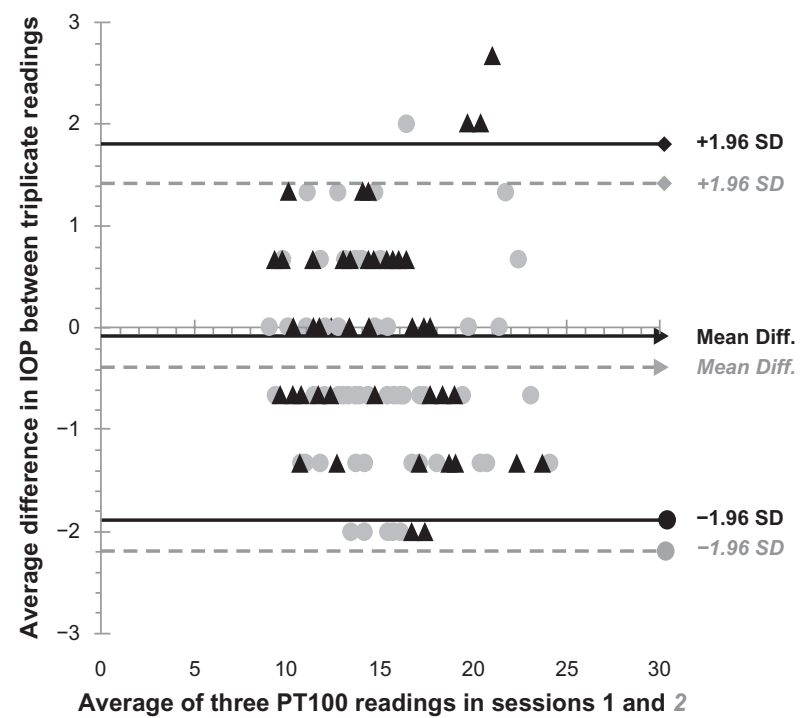

Figure 2 Intrasession variation of IOP with the PTI00 noncontact tonometer in sessions $I$ and 2 . The mean difference of triplicate IOP measurements for each subject; $95 \%$ limits of agreement are depicted by the three lines. Abbreviations: IOP, intraocular pressure; SD, standard deviation. 


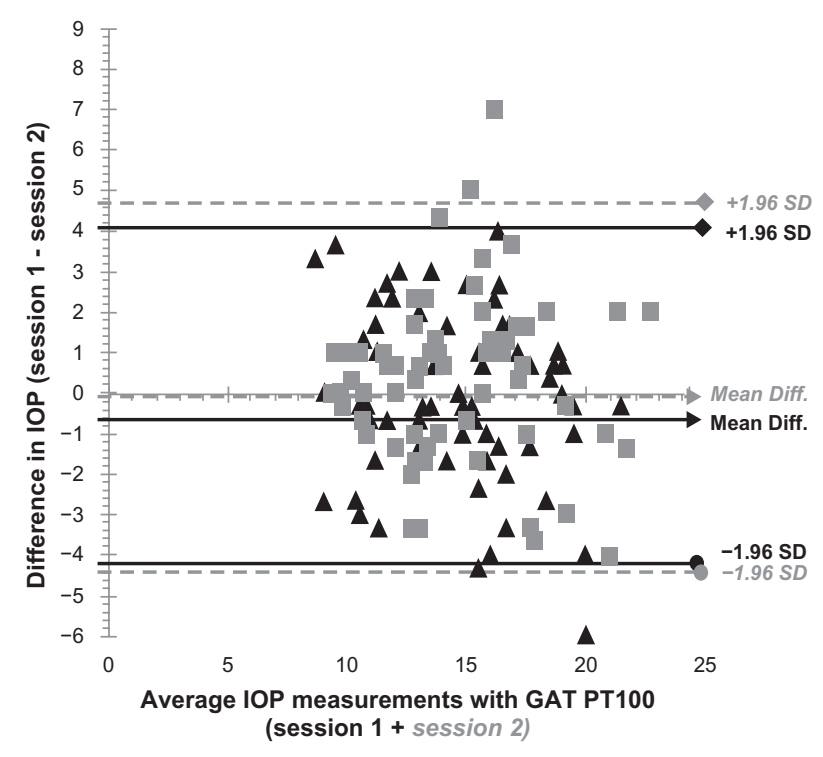

Figure 3 Test-retest variation of the Goldmann applanation tonometer and the Reichert PTI00 IOP measurements. The mean differences between both sessions, and the $95 \%$ limits of agreement are depicted by the three lines.

Abbreviations: IOP, intraocular pressure; SD, standard deviation.

for the GAT was $0.1 \mathrm{mmHg}$ superior to the PT100 in the first session, the PT100 was stronger in the second session. Overall, both instruments showed good repeatability, with the PT100 noncontact tonometer being more consistent in repeated measurement sessions. Two other studies ${ }^{5,6}$ have also shown similar repeatability coefficients for the GAT, $\pm 2.5 \mathrm{mmHg}^{5}$ and $3.70 \mathrm{mmHg}^{6}$ when compared with other noncontact tonometers. . $^{5,6}$

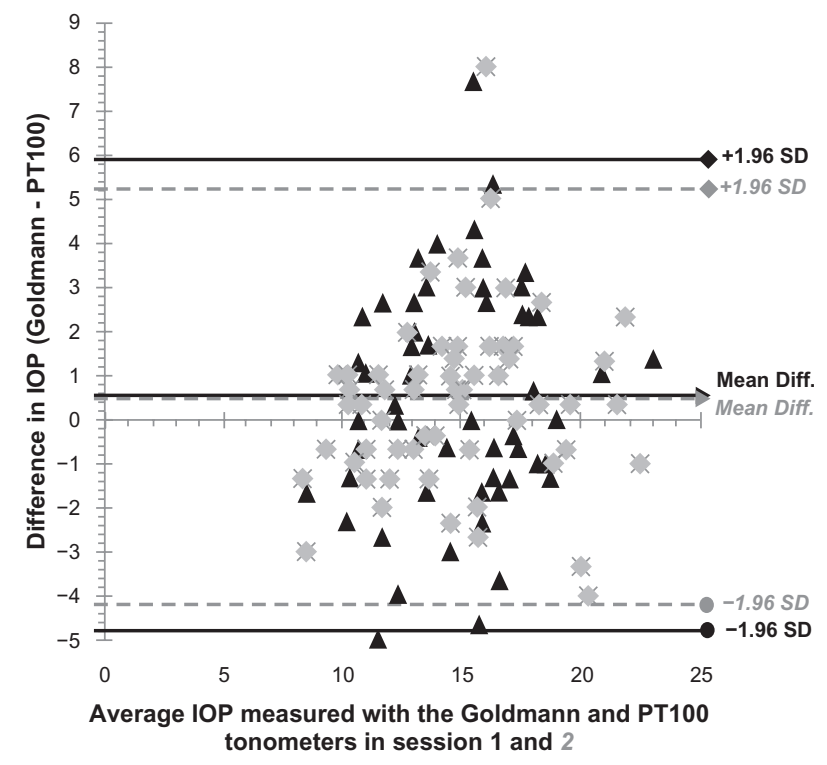

Figure 4 Differences in IOP, measured in I st and 2nd sessions, between the Goldmann tonometer and the PTI00 noncontact tonometer plot against their average, with the mean difference and $95 \%$ limits of agreement depicted by the three lines. Abbreviation: IOP, intraocular pressure.
Notable in this study is the identical within-session differences for the PT100 and the GAT for both sessions 1 and 2 . This means that both techniques have the same consistency in their measurements and can be interchanged in follow-up situations where a patient's previous IOP measurements had been taken with either one of the tonometers.

Clinical agreement between the noncontact tonometer and the GAT has been demonstrated in previous studies of normotensive subjects, ${ }^{5,6,29,30}$ with a propensity for the noncontact tonometer to return about $3 \mathrm{mmHg}$ higher IOP measurements than the $\mathrm{GAT}^{7,8,27,28}$ in subjects with IOPs up to $21 \mathrm{mmHg}$.

In one of the two previous published studies comparing the PT100 and the GAT, a strong agreement was observed between the two instruments. ${ }^{10,11}$ In the study by Salim et al $^{10}$ a close level of agreement in the normal range of IOPs was observed, with an increased variation as the magnitude of measurements increased. Ninety-eight subjects aged $62.6 \pm 13.8$ years had IOP measurements of $15.65 \pm 4.26 \mathrm{mmHg}$ and $15.98 \pm 5.48 \mathrm{mmHg}$ for the PT100 and the GAT, respectively. While their result is similar to ours, in comparative analysis of the agreement of the two instruments, our subjects' mean IOP measurements varied slightly from theirs (15 $\pm 3 \mathrm{mmHg}, \mathrm{PT} 100$ and GAT) and the mean IOP did not differ in the PT100 and the GAT. The large age variation of the subjects in the study by Salim et al could explain this discrepancy, because all of our subjects were in a younger age group ( $22 \pm 1$ years). This shows that the PT100 IOP measurement results are the same as for the GAT in normal healthy young subjects. ${ }^{10}$

In this study, the average IOP measured by both tonometers was the same, and no significant difference was found between the repeatability and reproducibility coefficients for the GAT and the PT100 noncontact tonometer.

The portability and ease of use of the PT100 noncontact tonometer makes it an ideal IOP measuring device for use in children during screening programs or in situations where a technician has to take IOP measurements in clinic. Just like previous noncontact tonometers, it has some limitations, ie, the need for adequate experience and expertise in order to take accurate measurements, and the absence of a headrest, making proper fixation a necessary requirement to acquire accurate measurements. Of consideration also is the effect of corneal properties on both the GAT and noncontact tonometers, with the latter being more affected by central corneal thickness than the GAT. ${ }^{25,26}$ Because of the relative low number of subjects, further studies on the reliability of the PT100 noncontact tonometer in subjects with higher IOPs 
is important and the effects of central corneal thickness on its measurements verified to confirm the report from Murase et $\mathrm{al}^{11}$ that the PT100 is more affected by variations in central corneal thickness than the GAT. However, further study in subjects with higher IOP and elderly patients is needed to confirm the agreement between the two methods.

In summary, this study has shown that the PT100 is an accurate and reliable method for assessing IOP in normotensive young subjects. The PT100 IOP readings are reproducible when two examiners take separate measurements, and are interchangeable with IOP measurements obtained with the GAT.

\section{Acknowledgment}

The author would like to thank Dr Kelechi Ogbuehi for his assistance with data collection for this study.

\section{Disclosure}

The author reports no conflicts of interest in this work.

\section{References}

1. The AGIS Investigators. The Advanced Glaucoma Intervention Study (AGIS): 7. The relationship between control of intraocular pressure and visual field deterioration. Am J Ophthalmol. 2000;130(4):429-440.

2. Litchter PR, Musch DC, Gillespie BW, et al; CIGTS Study Group. Interim clinical outcomes in the Collaborative Initial Glaucoma Treatment Study comparing initial treatment randomized to medications or surgery. Ophthalmology. 2001;108(11):1943-1953.

3. Gupta V, Sony P, Agarwal HC, Sihota R, Sharma A. Inter-instrument agreement and influence of central corneal thickness on measurements with Goldmann, pneumotonometer and noncontact tonometer in glaucomatous eyes. Indian J Ophthalmol. 2006;54(4):261-265.

4. Lam AK, Chan R, Lam CH. The validity of a new noncontact tonometer and its comparison with the Goldmann tonometer. Optom Vis Sci. 2004;81(8):601-605.

5. Almubrad TM, Ogbuehi KC. The effect of repeated applanation on subsequent IOP measurements. Clin Exp Optom. 2008;91(6):524-529.

6. García-Resúa C, Giráldez Fernández MJ, Yebra-Pimentel E, García-Montero S. Clinical evaluation of the Canon TX-10 noncontact tonometer in healthy eyes. Eur J Ophthalmol. 2010;20(3):523-530.

7. Regine F, Scuderi GL, Cesareo M, Ricci F, Cedrone C, Nucci C. Validity and limitations the Nidek NT-4000 non-contact tonometer: A clinical study. Ophthalmic Physiol Opt. 2006;26(1):33-39.

8. Ogbuehi KC. Assessment of the accuracy and reliability of the Topcon CT80 non-contact tonometer. Clin Exp Optom. 2006;89(5):310-314.

9. Van der Jagt LH, Jansonius NM. Three portable tonometers, the TGDc-01, the ICARE and the Tonopen XL, compared with each other and with Goldmann applanation tonometry. Ophthalmic Physiol Opt. 2005;25(5):429-435.

\section{Clinical Ophthalmology}

\section{Publish your work in this journal}

Clinical Ophthalmology is an international, peer-reviewed journal covering all subspecialties within ophthalmology. Key topics include: Optometry; Visual science; Pharmacology and drug therapy in eye diseases; Basic Sciences; Primary and Secondary eye care; Patient Safety and Quality of Care Improvements. This journal is indexed on Submit your manuscript here: http://www.dovepress.com/clinical-ophthalmology-journal
10. Salim S, Linn DJ, Echols JR, Netland PA. Comparison of intraocular pressure measurements with the portable PT100 noncontact tonometer and Goldmann applanation tonometry. Clin Ophthalmol. 2009;3: 341-344.

11. Murase H, Sawada A, Mochizuki K, Yamamoto T. Effects of corneal thickness on intraocular pressure measured with three different tonometers. Jpn J Ophthalmol. 2009;53(1):1-6.

12. Stocker FW. On changes in intraocular pressure after application of the tonometer; in the same eye and in the other eye. Am J Ophthalmol. 1958;45(2):192-196.

13. Moses RA. Repeated applanation tonometry. Ophthalmologica. 1961; 142:663-668.

14. Bynke HG, Schéle B. On the origin of the ocular pressure pulse. Ophthalmologica. 1967;153(1):29-36.

15. Leydhecker W. The intraocular pressure: Clinical aspects. Ann Ophthalmol. 1976;8(4):389-392.

16. Perkins ES. The ocular pulse. Curr Eye Res. 1981;1(1):19-23.

17. Krakau CE, Wilke K. On repeated tonometry. Acta Ophthalmol (Copenh). 1971;49(4):611-614.

18. Chauhan BC, Henson DB. Clinical evaluation of the non-contact tonometer Mark II. Am J Optom Physiol Opt. 1988;65(9):751-756.

19. Baudouin C, Gastaud P. Influence of topical anesthesia on tonometric values of intraocular pressure. Ophthalmologica. 1994;208(6): 309-313.

20. Almubrad TM, Ogbuehi KC. On repeated corneal applanation with the Goldmann and two non-contact tonometers. Clin Exp Optom. 2010;93(2):77-82.

21. Liu JH, Kripke DF, Twa MD, et al. Twenty-four-hour pattern of intraocular pressure in the aging population. Invest Ophthalmol Vis Sci. 1999;40(12):2912-2917.

22. Bland JM, Altman DG. Statistical methods for assessing agreement between two methods of clinical measurement. Lancet. 1986;1(8476): 307-310.

23. Bland JM, Altman DG. Measuring agreement in method comparison studies. Stat Methods Med Res. 1999;8(2):135-160.

24. Faul F, Erdfelder E, Lang AG, Buchner A. G*Power 3: A flexible statistical power analysis program for the social, behavioral, and biomedical sciences. Behav Res Methods. 2007;39(2):175-191.

25. Tonnu PA, Ho T, Newson T, et al. The influence of central corneal thickness and age on intraocular pressure measured by pneumotonometry, non-contact tonometry, the Tono-Pen XL, and Goldmann applanation tonometry. Br J Ophthalmol. 2005;89(7):851-854.

26. Ko YC, Liu CJ, Hsu WM. Varying effects of corneal thickness on intraocular pressure measurements with different tonometers. Eye (Lond). 2005;19(3):327-332.

27. Jorge J, Díaz-Rey JA, González-Méijome JM, Almeida JB, Parafita MA. Clinical performance of the Reichert AT550: A new non-contact tonometer. Ophthalmic Physiol Opt. 2002;22(6):560-564.

28. Jorge J, González-Méijome JM, Díaz-Rey JA, Almeida JB, Ribeiro P, Parafita MA. Clinical performance of non-contact tonometry by Reichert AT550 in glaucomatous patients. Ophthalmic Physiol Opt. 2003;23(6): 503-506.

29. Moreno-Montañés J, Gómez-Demmel E, Lajara-Blesa J, Aliseda-Pérez de Madrid D. Comparative study of three non-contact tonometers and the Goldmann tonometer. Ophthalmologica. 1994;208(3):115-118.

30. Sorensen PN. The noncontact tonometer: Clinical evaluation on normal and diseased eyes. Acta Ophthalmol (Copenh). 1975;53(4):513-521.

\section{Dovepress}

PubMed Central and CAS, and is the official journal of The Society of Clinical Ophthalmology (SCO). The manuscript management system is completely online and includes a very quick and fair peer-review system, which is all easy to use. Visit http://www.dovepress.com/ testimonials.php to read real quotes from published authors. 